\title{
Robust adaptive neural control for a class of non-affine nonlinear systems
}

\author{
Chao Shi, Xinmin Dong*, Jianping Xue, Yong Chen, Jianhui Zhi \\ Department of Flight control and Electrical engineering, Aeronautics and Astronautics Engineering College, Air Force Engineering University, \\ Xi'an, Shaanxi, 710038 China
}

\begin{abstract}
This paper addresses the adaptive neural tracking control problem for a class of uncertain non-affine nonlinear system with non-affine function being semi-bounded and possibly non-differentiable. Compared with traditional control schemes, the proposed scheme can be applied to a more general class of non-affine nonlinear system, and relaxes constraint conditions as follows: firstly, the assumption that non-affine function must be differentiable is canceled, and only a continuous condition for non-affine function is required to guarantee the controllability of the considered system, secondly, the assumption that non-affine function is completely bounded is relaxed, and the non-affine function is constrained by a semi-bounded condition with the bounds being unknown functions. Then, an adaptive neural tracking controller is designed based on an invariant set. In the control design process, minimal learning parameter (MLP) technique is used to reduce the number of adaptive parameters, and a smooth robust compensator is employed to circumvent the influences of approximation error and external disturbance. Furthermore, it is proven that all the closed-loop signals are semi-globally uniformly ultimately bounded. Finally, simulation examples are provided to demonstrate the effectiveness of the designed method.
\end{abstract}

Keywords: Adaptive control, Neural network, Non-affine nonlinear systems, Invariant set

\section{Introduction}

In recent years, a lot of efforts have been made in the field of approximation-based adaptive control schemes for uncertain nonlinear systems [1-11]. Due to the universal approximation, learning and adaptation abilities of neural network (NN), the adaptive controllers involving NN approximation have achieved fairly good stability and accurate trajectory tracking for unknown nonlinear dynamics. However, most of them are applicable to the relatively simple nonlinear systems in affine form. In practice, there exist many systems with non-affine structure, such as chemical reaction [12], dynamic model in pendulum control [13], etc. Owing to few choices of mathematical tools in handling nonaffine appearance of the control input, the adaptive control problem for non-affine nonlinear systems still remains challenging.

For non-affine nonlinear system, the main difficulty of controller design lies in that the control input does not appear in an affine form, and therefore, the control methods developed for affine nonlinear system cannot be directly employed to design controller for non-affine

Preprint submitted to Elsevier nonlinear system in general. To overcome the difficulty, some representative adaptive control schemes have been presented for non-affine nonlinear systems. In [14-16], by using a Taylor series expansion in the neighborhood of an unknown control input trajectory, the non-affine nonlinear system is transformed into an affine form, and then, adaptive fuzzy controllers are designed. However, the Taylor series expansion is only valid locally around a specified point. The mean value theorem is therefore employed to convert the non-affine function into a model of affine form since it is valid globally [1719]. Recently, the implicit function theorem is used to demonstrate the existence of an ideal controller that can achieve control objective, and then a NN or fuzzy system is used to construct this unknown ideal implicit controller for non-affine nonlinear systems [20-23]. In [2429], by combining the implicit function theorem with mean value theorem, adaptive NN controllers are proposed for non-affine nonlinear systems. However, it is worth noting that the common assumption of the aforementioned approaches is that non-affine function must be differentiable with respect to the control input. And to assure the controllability of the system, the partial

September 3, 2016 
derivative of non-affine function with respect to the control input is always assumed to be strictly positive or negative. Moreover, it is noticeable that for most of the existing approaches, the partial derivative of non-affine function with respect to the control input is constrained by both upper and lower bounds. Unfortunately, a priori knowledge of the plant dynamics is always difficult to be acquired for the bounds of the partial derivative of non-affine function, and it is well known that dead-zone and hysteresis exist widely in practical systems, which results in non-differentiable [7, 19, 30-34].

On the other hand, an approximation error will inevitably occur if a $\mathrm{NN}$ is used to approximate unknown nonlinear function of a given system [35-37], and external disturbances exist everywhere in actual systems, which may degrade the performance of the closed-loop control system, or even worse, deteriorate the stability. Recently, in [38], the tracking control problem for non-affine nonlinear systems is solved based on a selforganizing approximation approach. In [25], based on dynamic learning scheme, adaptive $\mathrm{NN}$ control is presented for a class of non-affine nonlinear systems. However, only the effect of NN approximation error is compensated in these methods. In [24], direct adaptive neural control is developed for a class of uncertain nonaffine nonlinear systems based on disturbance observer. A direct self-constructing neural controller is designed for a class of uncertain non-affine nonlinear systems [21]. An adaptive control scheme is proposed for uncertain non-affine nonlinear systems with input saturation using neural networks [7]. Although NN approximation error and external disturbance are considered in $[7,21,24]$, there are a large number of neural weights to be tuned online in these approaches, which increases the computational burden. Therefore, MLP technique is always employed to deal with the problem in some references on affine systems. In [39], combining DSC with MLP technique, a robust adaptive NN tracking control scheme has been proposed for a class of affine nonlinear systems.

Motivated by the above discussion, a novel adaptive neural tracking control scheme is proposed for a class of non-affine nonlinear system with non-affine function being semi-bounded and possibly non-differentiable. The main contributions of this paper are summarized as follows:

1) For uncertain non-affine function, the restrictive differential condition with respect to the control input is removed, and only a continuous condition is presented to guarantee the controllability of uncertain non-affine nonlinear system.

2) Different from most of the existing researches, the non-affine function is constrained by a semi-bounded condition with the bounds being unknown functions, which makes the controller design much more difficult. A compact set is then constructively applied in the controller design for non-affine nonlinear systems to deal with the difficulty.

3) By introducing a robust term, the effects of NN approximation error and external disturbance are compensated such that the proposed approach is able to handle a certain degree of uncertainties. Furthermore, MLP technique is employed to reduce the number of adaptive parameters.

This paper is organized as follows. In Section 2, the problem formulation and preliminaries are provided. The adaptive neural tracking control design approach and stability analysis of the closed-loop system are presented in Section 3. Two simulation examples are given to demonstrate the effectiveness of the proposed method in Section 4. We conclude the work in Section 5.

\section{Problem formulation and preliminaries}

\subsection{Problem formulation}

Consider a class of uncertain SISO non-affine nonlinear systems as follows

$$
\left\{\begin{array}{l}
\dot{x}_{i}=x_{i+1}, \quad i=1,2, \ldots, n-1 \\
\dot{x}_{n}=f(x, u)+d(t) \\
y=x_{1}
\end{array}\right.
$$

where $x=\left[x_{1}, x_{2}, \ldots, x_{n}\right]^{T} \in R^{n}, u \in R$ and $y \in R$ are the system state, control input and output, respectively; $f(x, u)$ is an unknown non-affine continuous function, and $d(t)$ is an unknown external disturbance.

The control objective of this paper is to design an adaptive neural tracking controller for system (1) such that the output $y$ follows the desired trajectory $y_{d}$ and all the signals in the closed-loop system remain semiglobally uniformly ultimately bounded in the presence of external disturbance.

Throughout this paper, we make the following assumptions and lemmas.

Assumption 1: Define a function $L(x, u)$ as follows

$$
L(x, u)=f(x, u)-f(x, 0)
$$

And for all $x$ and $u$, there exist unknown constants $b_{1}$ and $b_{2}$ such that

$$
\begin{cases}L(x, u) \geq H_{1}(x) u+b_{1}, & u \geq 0 \\ L(x, u) \leq H_{2}(x) u+b_{2}, & u<0\end{cases}
$$


where $H_{1}(x)$ and $H_{2}(x)$ are unknown continuous functions satisfying $H_{1}(x)>0, H_{2}(x)>0$.

Remark 1: It is noticeable that $f(x, u)$ is commonly assumed to satisfy $0<g_{1} \leq \partial f(x, u) / \partial u \leq g_{2},\left(g_{1}, g_{2} \in\right.$ $R)$ such as in [6], [21], [22], which can be seen as the controllability condition of their systems. However, in this paper, Assumption 1 is used to guarantee the controllability of system (1), while the assumption that nonaffine function must be differentiable is canceled. Furthermore, by the partial integration for $\partial f(x, u) / \partial u$ with respect to $u$ in [6], [21], [22], we can obtain

$$
\begin{cases}g_{1} u \leq f(x, u)-f(x, 0) \leq g_{2} u, & u \geq 0 \\ g_{2} u \leq f(x, u)-f(x, 0) \leq g_{1} u, & u<0\end{cases}
$$

Then, compared with (4), Assumption 1 relaxes the constraint condition as continuous and semi-bounded condition, and is a reasonable relaxation of (4). Therefore, Assumption 1 is valid in this paper. Moreover, due to the presence of unknown continuous function $H_{1}(x)$ and $H_{2}(x)$, the bounds of semi-bounded condition are also unknown continuous functions, which makes the design of system controller more difficult. Thus, a compact set is introduced later to overcome this difficulty.

Assumption 2: For $\forall t>0$, there exists unknown positive constant $d^{*}$ such that $|d(t)| \leq d^{*}$.

Assumption 3: The desired trajectory $y_{d}$ is bounded and sufficiently smooth, namely, there exist $\dot{y}_{d}, \ddot{y}_{d}, \ldots, y_{d}^{(n)}$ included in the compact set $\Omega_{0}:=$ $\left\{\left(y_{d}, \dot{y}_{d}, \ldots, y_{d}^{(n)}\right): \sum_{i=0}^{n}\left(y_{d}^{(i)}\right)^{2} \leq \mathrm{A}_{0}\right\}$, where $y_{d}^{(0)}$ denotes $y_{d} ; \mathrm{A}_{0}$ is an unknown positive constant. For simplicity, we define $x_{d}=\left[y_{d}, \dot{y}_{d}, \ldots, y_{d}^{(n-1)}\right]^{T}$ in this paper.

Lemma 1 [40]: Consider the dynamic system in the form of

$$
\dot{\chi}(t)=-\varpi \chi(t)+\vartheta v(t)
$$

where $\varpi$ and $\vartheta$ are positive constants and $v(t)$ is a positive function. Given any bounded initial condition $\chi(0) \geq 0$, then $\chi(t) \geq 0$ holds for all $\forall t \geq 0$.

Lemma 2 [41]: The hyperbolic tangent function $\tanh (\cdot)$ is continuous and differentiable, and it fulfils that for $\forall q \in R$ and $\forall \varsigma>0$

$$
0 \leq|q|-q \tanh \left(\frac{q}{\varsigma}\right) \leq 0.2785 \varsigma
$$

Lemma 3 (Young's inequality [42]): For $\forall(x, y) \in R^{2}$, the following inequality holds

$$
x y \leq \frac{\varepsilon^{p}}{p}|x|^{p}+\frac{1}{q \varepsilon^{q}}|y|^{q}
$$

where $\varepsilon>0, p>1, q>1$ and $(p-1)(q-1)=1$. We choose $p=q=2$ in this paper.

\subsection{RBF neural network approximation}

In this paper, the radial basis function neural network (RBFNN) will be applied to approximate the continuous function $g(Z): R^{n} \rightarrow R$

$$
g_{n n}(Z)=W^{T} \phi(Z)
$$

where $Z=\left[Z_{1}, Z_{2}, \ldots, Z_{n}\right]^{T} \in \Omega_{Z} \subset R^{n}$ is the input vector; $W=\left[W_{1}, W_{2}, \ldots, W_{l}\right]^{T} \in R^{l}$ is the weight vector; $l$ denotes the NN node number; and $\phi(Z)=$ $\left[\phi_{1}(Z), \phi_{2}(Z), \ldots, \phi_{l}(Z)\right]^{T} \in R^{l}$, with $\phi_{i}(Z)$ being chosen as the commonly used Gaussian functions as follows

$$
\phi_{i}(Z)=\exp \left[\frac{-\left(Z-\mu_{i}\right)^{T}\left(Z-\mu_{i}\right)}{\sigma_{i}^{2}}\right], \quad i=1,2, \ldots ., l
$$

where $\mu_{i}=\left[\mu_{i 1}, \mu_{i 2}, \ldots, \mu_{i n}\right]^{T}$ is the center of the receptive field and $\sigma_{i}$ is the width of the Gaussian function.

As proven in [43], by choosing enough neural nodes, the neural network (8) can approximate any continuous function over a compact set $\Omega_{Z} \subset R^{n}$ with arbitrary accuracy.

$$
g(Z)=W^{* T} \phi(Z)+\varepsilon(Z), \forall Z \in \Omega_{Z}
$$

where $W^{*}$ is the ideal constant weight vector, and $\varepsilon(Z)$ is the approximation error which satisfies $|\varepsilon(Z)| \leq \varepsilon^{*}$ with $\varepsilon^{*}>0$ being an unknown constant.

For simplicity, in this paper, $\varepsilon$ denotes $\varepsilon(Z),\|x\|$ denotes the 2-norm of vector $x$, and define $\tilde{\delta}=\delta-\hat{\delta}$ with $\hat{\delta}$ being the estimate of unknown constant $\delta$ and $\tilde{\theta}=\theta-\hat{\theta}$ with $\hat{\theta}$ being the estimate of unknown constant $\theta$.

\section{Controller methodology}

\subsection{Error dynamics and system transformation}

Define the system state tracking error as

$$
e=\left[e_{1}, e_{2}, \ldots, e_{n}\right]^{T}, \quad e_{i}=x_{i}-y_{d}^{(i-1)}, e \in R^{n}
$$

From (11), we have

$$
x=e+\left[y_{d}, \dot{y}_{d}, \ldots, y_{d}^{(n-1)}\right]^{T}
$$

Define the filtering error as

$$
s=\left(\frac{d}{d t}+q\right)^{n-1} e_{1}=\left[\lambda_{1}, \lambda_{2}, \ldots, \lambda_{n-1}, 1\right] e, s \in R
$$

where $\lambda_{i}=C_{n-1}^{i-1} q^{n-i},(i=1, \ldots, n-1), C_{n-1}^{i-1}$ denotes the sign of permutations and combinations defined as $C_{n-1}^{i-1}=\frac{(n-1) !}{(i-1) !(n-i) !}$, and $q$ is a positive design 
parameter. For the definition of (13), the following Lemma 4 is introduced for the control design.

Lemma 4 [44]: The definition (13) for $s$ have the following properties:

i) If $s=0$, then $\left[\lambda_{1}, \lambda_{2}, \ldots, \lambda_{n-1}, 1\right] e=0$, which is a hyperplane in $R^{n}$. And the tracking error $e_{1}$ converges to zero asymptotically on this hyperplane.

ii) If $|s(t)| \leq B$ for $\forall t \geq 0$ with $B$ being a positive constant, then $e(t)$ will converge to the following compact set

$$
\Omega_{e}=\left\{e|| e_{i} \mid \leq 2^{i-1} q^{i-n} B, i=1,2, \ldots, n\right\}, \forall t \geq T_{0}
$$

where $T_{0} \geq 0$ is a computable constant.

According to Lemma 4, in order to confine the tracking error $e$, we need to guarantee the convergence of the filtering error $s$. By recalling (1), (11) and (13), the dynamics of filtering error $s$ can be written as

$$
\begin{aligned}
\dot{s} & =\left[0, \lambda_{1}, \lambda_{2}, \ldots, \lambda_{n-1}\right] e+\dot{e}_{n} \\
& =f(x, u)+d(t)-y_{d}^{(n)}+\sum_{i=1}^{n-1} \lambda_{i} e_{i+1} \\
& =f(x, u)+d(t)+\Phi
\end{aligned}
$$

where $\Phi=-y_{d}^{(n)}+\sum_{i=1}^{n-1} \lambda_{i} e_{i+1}$.

Consider the following Lyapunov function candidate

$$
V_{s}=\frac{1}{2} s^{2}
$$

Define a compact set $\Omega_{s}=\left\{s \mid V_{s} \leq p\right\}$, where $p$ is an arbitrary positive constant. By noting (13), we can know that $\Omega_{s}$ is equivalent to

$$
\Omega_{s}=\left\{\left(e_{1}, e_{2}, \ldots, e_{n}\right) \mid\left(\sum_{i=1}^{n-1} \lambda_{i} e_{i}+e_{n}\right)^{2} \leq 2 p\right\}
$$

From (12), it is easily seen that $x$ can be viewed as a continuous function with respect to $e_{1}, e_{2}, \ldots, e_{n}$, $y_{d}, \dot{y}_{d}, \ldots, y_{d}^{(n-1)}$. Therefore, $L(x, 0), H_{1}(x)$ and $H_{2}(x)$ are the continuous functions of $e_{1}, e_{2}, \ldots, e_{n}, y_{d}, \dot{y}_{d}, \ldots, y_{d}^{(n-1)}$. Then, they can be expressed as follows

$$
\left\{\begin{array}{l}
L(x, 0)=\kappa_{L_{0}}\left(e_{1}, e_{2}, \ldots, e_{n}, y_{d}, \dot{y}_{d}, \ldots, y_{d}^{(n-1)}\right) \\
H_{1}(x)=\kappa_{H_{1}}\left(e_{1}, e_{2}, \ldots, e_{n}, y_{d}, \dot{y}_{d}, \ldots, y_{d}^{(n-1)}\right) \\
H_{2}(x)=\kappa_{H_{2}}\left(e_{1}, e_{2}, \ldots, e_{n}, y_{d}, \dot{y}_{d}, \ldots, y_{d}^{(n-1)}\right)
\end{array}\right.
$$

where $\kappa_{L_{0}}(\cdot), \kappa_{H_{1}}(\cdot)$ and $\kappa_{H_{2}}(\cdot)$ are unknown continuous functions.

Since $\Omega_{s}$ and $\Omega_{0}$ are compact sets, $\Omega_{s} \times \Omega_{0}$ is also a compact set. It is shown from (18) that all the variables of $\kappa_{L_{0}}(\cdot), \kappa_{H_{1}}(\cdot)$ and $\kappa_{H_{2}}(\cdot)$ are included in the compact set $\Omega_{s} \times \Omega_{0}$. Therefore, $\kappa_{L_{0}}(\cdot), \kappa_{H_{1}}(\cdot)$ and $\kappa_{H_{2}}(\cdot)$ have maximums and minimums on $\Omega_{s} \times \Omega_{0}$. Namely, based on $\Omega_{s} \times \Omega_{0}$, we have

$$
\left\{\begin{array}{l}
|L(x, 0)|=\kappa_{L_{0}}\left(e_{1}, e_{2}, \ldots, e_{n}, y_{d}, \dot{y}_{d}, \ldots, y_{d}^{(n-1)}\right) \leq N \\
H_{1}(x)=\kappa_{H_{1}}\left(e_{1}, e_{2}, \ldots, e_{n}, y_{d}, \dot{y}_{d}, \ldots, y_{d}^{(n-1)}\right) \geq H_{1 m}>0 \\
H_{2}(x)=\kappa_{H_{2}}\left(e_{1}, e_{2}, \ldots, e_{n}, y_{d}, \dot{y}_{d}, \ldots, y_{d}^{(n-1)}\right) \geq H_{2 m}>0
\end{array}\right.
$$

where $N$ is the maximum for $\kappa_{L_{0}}(\cdot)$ on $\Omega_{s} \times \Omega_{0} ; H_{1 m}$ is the minimum for $\kappa_{H_{1}}(\cdot)$ on $\Omega_{s} \times \Omega_{0} ; H_{2 m}$ is the minimum for $\kappa_{H_{2}}(\cdot)$ on $\Omega_{s} \times \Omega_{0}$.

Remark 2: In this paper, we will design the adaptive controller $u$ to be the continuous function of $\hat{\theta}, \hat{\delta}, e_{1}, e_{2}, \ldots, e_{n}, y_{d}, \dot{y}_{d}, \ldots, y_{d}^{(n)}$, and it can be expressed as follows

$$
u=u\left(\hat{\theta}, \hat{\delta}, e_{1}, e_{2}, \ldots, e_{n}, y_{d}, \dot{y}_{d}, \ldots, y_{d}^{(n)}\right)
$$

Furthermore, by noting (12), it can be seen that $x$ is a continuous function with respect to $e_{1}, e_{2}, \ldots, e_{n}, y_{d}$, $\dot{y}_{d}, \ldots, y_{d}^{(n-1)}$. Then, we can know that $L(x, u)$ is a continuous function with respect to $u$ and $x$, and it can be expressed as follows

$$
L(x, u)=\kappa_{L}\left(\hat{\theta}, \hat{\delta}, e_{1}, e_{2}, \ldots, e_{n}, y_{d}, \dot{y}_{d}, \ldots, y_{d}^{(n)}\right)
$$

where $\kappa_{L}(\cdot)$ is an unknown continuous function.

Considering $f(x, 0)$ is an unknown function, we use a RBFNN to approximate it over a compact set $\Omega_{x}$ as follows

$$
f(x, 0)=W^{* T} \phi(x)+\varepsilon
$$

where $W^{*}$ is the unknown ideal weight vector, and $\varepsilon$ is the approximation error satisfying $|\varepsilon| \leq \varepsilon^{*}$ with $\varepsilon^{*}>0$. To reduce the number of adaptive parameters, based on MLP technique, we define the constant

$$
\theta=H_{m}^{-1}\left\|W^{*}\right\|^{2}
$$

where $H_{m}=\min \left\{H_{1 m}, H_{2 m}\right\}$ is an unknown positive constant.

Consider the following Lyapunov function

$$
V=V_{s}+\frac{H_{m}}{2 \gamma} \tilde{\delta}^{2}+\frac{H_{m}}{2 \beta} \tilde{\theta}^{2}
$$

where $\gamma>0$ and $\beta>0$ are design parameters, and $\delta=$ $H_{m}^{-1}\left(\Delta^{*}+\varepsilon^{*}+d^{*}\right), \Delta^{*}=\max \left\{N,\left|b_{1}\right|,\left|b_{2}\right|\right\}$ are unknown positive constants.

Define a compact set $\Omega_{1}:=\{(\hat{\theta}, \hat{\delta}, s) \mid V \leq p\}$. Noting (13) and (24), $\Omega_{1}$ can be rewritten as

$$
\begin{aligned}
\Omega_{1}= & \left\{\left(\hat{\theta}, \hat{\delta}, e_{1}, e_{2}, \ldots, e_{n}\right) \mid\right. \\
& \left.\left(\sum_{i=1}^{n-1} \lambda_{i} e_{i}+e_{n}\right)^{2}+\frac{H_{m}}{\gamma} \tilde{\delta}^{2}+\frac{H_{m}}{\beta} \tilde{\theta}^{2} \leq 2 p\right\}
\end{aligned}
$$


Since the sets $\Omega_{0} \in R^{n+1}$ and $\Omega_{1} \in R^{n+2}$ are compact, $\Omega_{2}=\Omega_{1} \times \Omega_{0} \in R^{2 n+3}$ is also a compact set. From (21), we can see that $\Omega_{2}$ includes all the variables of continuous function $\kappa_{L}(\cdot)$. Therefore, $\kappa_{L}(\cdot)$ has a maximum $M$ on $\Omega_{2}$, namely, on the compact set $\Omega_{2}$, we have

$$
|L(x, u)| \leq M
$$

From (26), we can obtain

$$
-M \leq L(x, u) \leq M
$$

Remark 3: It should be pointed out that (26) is satisfied on the set $\Omega_{2}$ and (19) is also satisfied on the set $\Omega_{2}$ because $\Omega_{2} \subset \Omega_{s} \times \Omega_{0} \times R^{2}$. Since that compact set $\Omega_{1}$ can be written as $\Omega_{1}=\left\{\left(\hat{\theta}, \hat{\delta}, e_{1}, e_{2}, \ldots, e_{n}\right) \mid V \leq p\right\}$ by noting (24), we know that the initial position of $\left(\hat{\theta}, \hat{\delta}, e_{1}, e_{2}, \ldots, e_{n}, y_{d}, \dot{y}_{d}, \ldots, y_{d}^{(n)}\right)$ is in $\Omega_{2}$ according to the initial condition $V(0) \leq p$. Therefore, similar as [45], if $\dot{V} \leq 0$ for $V=p$ based on the inequalities (19) and (26), then we can conclude that $V \leq p$ for $\forall t>0$, namely, $\Omega_{2}$ is an invariant set, and hence the stability of the whole system is achieved. Thus, in the following part, the stability of system (1) will be proven on the basis of (19) and (26). It also should be mentioned that (19) and (26) are satisfied based on $\Omega_{2}$, though $L(x, u)$ is completely bounded in these inequalities, which does not contradict with Assumption 1.

It follows from (3) and (27) that

$$
\left\{\begin{array}{l}
H_{1}(x) u+b_{1} \leq L(x, u) \leq M, \quad u \geq 0 \\
-M \leq L(x, u) \leq H_{2}(x) u+b_{2}, \quad u<0
\end{array}\right.
$$

which can be rewritten as

$$
\left\{\begin{array}{l}
H_{1}(x) u+b_{1} \leq L(x, u) \leq \frac{M-b_{1}}{u} u+b_{1}, \quad u>0 \\
\frac{-M-b_{2}}{u} u+b_{2} \leq L(x, u) \leq H_{2}(x) u+b_{2}, \quad u<0
\end{array}\right.
$$

Using (29), one has

$$
\left\{\begin{array}{l}
H_{1}(x) u+b_{1} \leq \frac{M-b_{1}}{u} u+b_{1}, \quad u>0 \\
\frac{-M-b_{2}}{u} u+b_{2} \leq H_{2}(x) u+b_{2}, \quad u<0
\end{array}\right.
$$

Define $h_{1}(u)=\left(M-b_{1}\right) / u$ and $h_{2}(u)=\left(-M-b_{2}\right) / u$. From (30), we can know

$$
\begin{cases}h_{1}(u) \geq H_{1}(x) \geq H_{1 m}, & \text { for } \forall u>0 \\ h_{2}(u) \geq H_{2}(x) \geq H_{2 m}, & \text { for } \forall u<0\end{cases}
$$

Noting (29) and the definition of $h_{1}(u)$ and $h_{2}(u)$, we have

$$
\left\{\begin{array}{l}
H_{1}(x) u+b_{1} \leq L(x, u) \leq h_{1}(u) u+b_{1}, u>0 \\
h_{2}(u) u+b_{2} \leq L(x, u) \leq H_{2}(x) u+b_{2}, u<0
\end{array}\right.
$$

Then, it can be learned from (32) that there exist functions $\alpha_{1}(t)$ and $\alpha_{2}(t)$ taking values in the closed interval $[0,1]$ and satisfying

$$
\left\{\begin{aligned}
L(x, u)= & \alpha_{1}(t)\left(H_{1}(x) u+b_{1}\right) \\
& +\left(1-\alpha_{1}(t)\right)\left(h_{1}(u) u+b_{1}\right), u>0 \\
L(x, u)= & \alpha_{2}(t)\left(h_{2}(u) u+b_{2}\right) \\
& +\left(1-\alpha_{2}(t)\right)\left(H_{2}(x) u+b_{2}\right), u<0
\end{aligned}\right.
$$

To facilitate the controller design, define the piecewise functions $h(x, u, t)$ and $\Delta(t)$ as follows

$$
\begin{aligned}
h(x, u, t) & = \begin{cases}\alpha_{1}(t) H_{1}(x)+\left(1-\alpha_{1}(t)\right) h_{1}(u), & u>0 \\
H_{1}(x), & u=0 \\
\alpha_{2}(t) h_{2}(u)+\left(1-\alpha_{2}(t)\right) H_{2}(x), & u<0\end{cases} \\
\Delta(t) & = \begin{cases}b_{1}, & u>0 \\
L(x, 0), & u=0 \\
b_{2}, & u<0\end{cases}
\end{aligned}
$$

Utilizing (31) and (34), we have

$$
h(x, u, t) \geq H_{m}>0
$$

According to (19) and (34), we can get

$$
\Delta^{*} \geq|\Delta(t)|>0
$$

From (33) and (34), $L(x, u)$ can be rewritten as

$$
L(x, u)=h(x, u, t) u+\Delta(t)
$$

Then, with the help of (37) and (2), system (1) can be rewritten in the form of

$$
\left\{\begin{array}{l}
\dot{x}_{i}=x_{i+1}, \quad i=1,2 \ldots, n-1 \\
\dot{x}_{n}=h(x, u, t) u+\Delta(t)+f(x, 0)+d(t) \\
y=x_{1}
\end{array}\right.
$$

Remark 4: It can be seen from (28)-(38) that the control gain function $h(x, u, t)$ is a positive function, namely, the control direction is positive, which guarantees the controllability of system (1) without the condition $\partial f(x, u) / \partial u$ existing. Furthermore, from the abovementioned derivation process, it can be observed that (38) hold based on Assumption 1. Therefore, Assumption 1 is the controllability condition of system (1).

\subsection{Controller design}

In this subsection, an adaptive neural tracking controller is proposed for system (1). To facilitate readers' comprehension, the overall block diagram of the proposed control scheme is presented in Fig.1. 


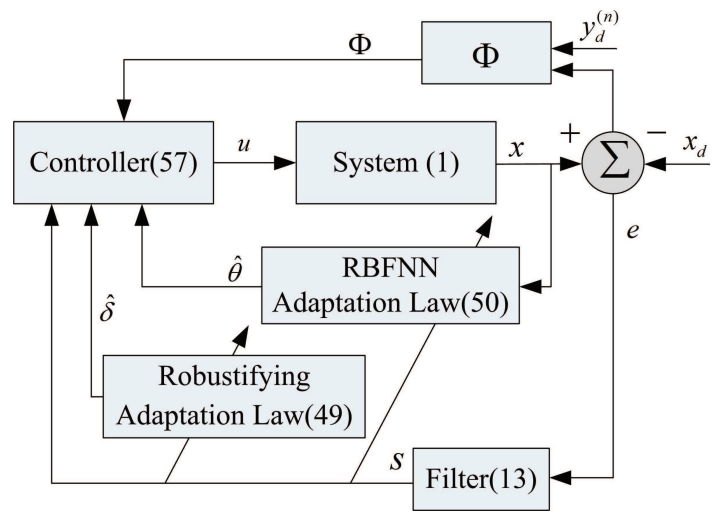

Figure 1: Block diagram of the adaptive neural control system

To begin with this design, the time derivative of $V_{s}$ along (15) is

$$
\dot{V}_{s}=s f(x, u)+s d(t)+s \Phi
$$

According to Assumption 2, we obtain

$$
\dot{V}_{s} \leq s f(x, u)+|s| d^{*}+s \Phi
$$

Noting (2), we can rewrite (40) as

$$
\dot{V}_{s} \leq s[L(x, u)+f(x, 0)]+|s| d^{*}+s \Phi
$$

Substituting (22) into (41) yields

$$
\begin{aligned}
\dot{V}_{s} & \leq s L(x, u)+s\left(W^{* T} \phi(x)+\varepsilon\right)+|s| d^{*}+s \Phi \\
& \leq s L(x, u)+s W^{* T} \phi(x)+|s| \varepsilon^{*}+|s| d^{*}+s \Phi
\end{aligned}
$$

Applying Lemma 3, we get

$$
s W^{* T} \phi(x) \leq \frac{s^{2}\left\|W^{*}\right\|^{2}}{2 a^{2}} \phi^{T}(x) \phi(x)+\frac{a^{2}}{2}
$$

Substituting (43) into (42), one has

$$
\begin{aligned}
\dot{V}_{s} \leq & s L(x, u)+\frac{s^{2}\left\|W^{*}\right\|^{2}}{2 a^{2}} \phi^{T}(x) \phi(x)+\frac{a^{2}}{2} \\
& +s \Phi+|s|\left(\varepsilon^{*}+d^{*}\right)
\end{aligned}
$$

It follows from (24) and (44) that the time derivative of $V$ is

$$
\begin{aligned}
\dot{V} \leq & s L(x, u)+\frac{s^{2}\left\|W^{*}\right\|^{2}}{2 a^{2}} \phi^{T}(x) \phi(x)+\frac{a^{2}}{2}+s \Phi \\
& +|s|\left(\varepsilon^{*}+d^{*}\right)+\frac{H_{m}}{\gamma} \tilde{\delta} \dot{\tilde{\delta}}+\frac{H_{m}}{\beta} \tilde{\theta} \dot{\tilde{\theta}}
\end{aligned}
$$

Substituting (37) into (45), we have

$$
\begin{aligned}
\dot{V} \leq & s h(x, u, t) u+s \Delta(t)+\frac{s^{2}\left\|W^{*}\right\|^{2}}{2 a^{2}} \phi^{T}(x) \phi(x)+\frac{a^{2}}{2} \\
& +s \Phi+|s|\left(\varepsilon^{*}+d^{*}\right)+\frac{H_{m}}{\gamma} \tilde{\delta} \dot{\tilde{\delta}}+\frac{H_{m}}{\beta} \tilde{\theta} \dot{\tilde{\theta}}
\end{aligned}
$$

Noting $\delta=H_{m}^{-1}\left(\Delta^{*}+\varepsilon^{*}+d^{*}\right)$ and using (36), we can further have

$$
\begin{aligned}
\dot{V} \leq & s h(x, u, t) u+\frac{s^{2}\left\|W^{*}\right\|^{2}}{2 a^{2}} \phi^{T}(x) \phi(x)+\frac{a^{2}}{2}+s \Phi \\
& +|s|\left(\Delta^{*}+\varepsilon^{*}+d^{*}\right)+\frac{H_{m}}{\gamma} \tilde{\delta} \tilde{\tilde{\delta}}+\frac{H_{m}}{\beta} \tilde{\theta} \dot{\tilde{\theta}} \\
\leq & s h(x, u, t) u+\frac{s^{2}\left\|W^{*}\right\|^{2}}{2 a^{2}} \phi^{T}(x) \phi(x)+\frac{a^{2}}{2}+s \Phi \\
& +|s| H_{m} \delta+\frac{H_{m}}{\gamma} \tilde{\delta} \dot{\tilde{\delta}}+\frac{H_{m}}{\beta} \tilde{\theta} \dot{\tilde{\theta}}
\end{aligned}
$$

Using $\dot{\tilde{\delta}}=-\dot{\hat{\delta}}, \dot{\tilde{\theta}}=-\dot{\hat{\theta}}$ and $\theta=H_{m}^{-1}\left\|W^{*}\right\|^{2}$, we have

$$
\begin{gathered}
\dot{V} \leq s h(x, u, t) u+\frac{s^{2} H_{m} \theta}{2 a^{2}} \phi^{T}(x) \phi(x)+\frac{a^{2}}{2} \\
+s \Phi+|s| H_{m} \delta-\frac{H_{m}}{\gamma} \tilde{\delta} \dot{\hat{\delta}}-\frac{H_{m}}{\beta} \tilde{\theta} \dot{\hat{\theta}}
\end{gathered}
$$

Choose the adaptive laws as follows

$$
\begin{aligned}
& \dot{\hat{\delta}}=\gamma s \tanh \left(\frac{s}{\varsigma}\right)-v_{1} \gamma \hat{\delta} \\
& \dot{\hat{\theta}}=\frac{\beta s^{2}}{2 a^{2}} \phi^{T}(x) \phi(x)-v_{2} \beta \hat{\theta}
\end{aligned}
$$

where $\varsigma>0, v_{1}>0, v_{2}>0$ and $a>0$ are design parameters.

Remark 5: Note that (49) and (50) satisfy the conditions of Lemma 1, therefore we have $\hat{\theta}(t) \geq 0, \hat{\delta}(t) \geq 0$ for $\forall t>0$ by choosing $\hat{\theta}(0) \geq 0, \hat{\delta}(0) \geq 0$, respectively. This property is very important and useful in our design. Furthermore, to make use of the property of Lemma 2 later, we introduce the hyperbolic tangent function $\tanh (\cdot)$ in $(49)$.

Substituting (49) and (50) into (48) yields

$$
\begin{aligned}
\dot{V} \leq & s h(x, u, t) u+\frac{s^{2} H_{m} \theta}{2 a^{2}} \varphi^{T}(x) \varphi(x)+\frac{a^{2}}{2} \\
& +|s| H_{m} \delta-\frac{H_{m}}{\gamma} \tilde{\delta}\left[\gamma s \tanh \left(\frac{s}{\varsigma}\right)-v_{1} \gamma \hat{\delta}\right] \\
& +s \Phi-\frac{H_{m}}{\beta} \tilde{\theta}\left[\frac{\beta s^{2}}{2 a^{2}} \varphi^{T}(x) \varphi(x)-v_{2} \beta \hat{\theta}\right]
\end{aligned}
$$


Using $\tilde{\delta}=\delta-\hat{\delta}$ and $\tilde{\theta}=\theta-\hat{\theta}$, we can further obtain

$$
\begin{aligned}
\dot{V} \leq & s h(x, u, t) u+\frac{s^{2} H_{m} \hat{\theta}}{2 a^{2}} \phi^{T}(x) \phi(x)+\frac{a^{2}}{2} \\
& +H_{m} \delta\left[|s|-s \tanh \left(\frac{s}{\varsigma}\right)\right]+s H_{m} \hat{\delta} \tanh \left(\frac{s}{\varsigma}\right) \\
& +\left[|s \Phi|-s \Phi \tanh \left(\frac{s \Phi}{\varsigma}\right)\right]+s \Phi \tanh \left(\frac{s \Phi}{\varsigma}\right) \\
& +v_{1} H_{m} \tilde{\delta} \hat{\delta}+v_{2} H_{m} \tilde{\theta} \hat{\theta}
\end{aligned}
$$

From Lemma 2, we can further have

$$
\begin{aligned}
\dot{V} \leq & s h(x, u, t) u+\frac{s^{2} H_{m} \hat{\theta}}{2 a^{2}} \phi^{T}(x) \phi(x)+s H_{m} \hat{\delta} \tanh \left(\frac{s}{\varsigma}\right) \\
& +s \Phi \tanh \left(\frac{s \Phi}{\varsigma}\right)+0.2785 \varsigma\left(H_{m} \delta+1\right) \\
& +v_{1} H_{m} \tilde{\delta} \hat{\delta}+v_{2} H_{m} \tilde{\theta} \hat{\theta}+\frac{a^{2}}{2}
\end{aligned}
$$

Using $\hat{\delta}=\delta-\tilde{\delta}$ and $\hat{\theta}=\theta-\tilde{\theta}$, we can rewrite (53) as

$$
\begin{aligned}
\dot{V} \leq & s h(x, u, t) u+\frac{s^{2} H_{m} \hat{\theta}}{2 a^{2}} \phi^{T}(x) \phi(x)+s H_{m} \hat{\delta} \tanh \left(\frac{s}{\varsigma}\right) \\
& +s \Phi \tanh \left(\frac{s \Phi}{\varsigma}\right)+0.2785 \varsigma\left(H_{m} \delta+1\right) \\
& +v_{1} H_{m}\left(\tilde{\delta} \delta-\tilde{\delta}^{2}\right)+v_{2} H_{m}\left(\tilde{\theta} \theta-\tilde{\theta}^{2}\right)
\end{aligned}
$$

Applying Lemma 3 to $\tilde{\delta} \delta$ and $\tilde{\theta} \theta$, we can obtain

$$
\left\{\begin{array}{l}
\tilde{\delta} \delta \leq \frac{\delta^{2}}{2}+\frac{\tilde{\delta}^{2}}{2} \\
\tilde{\theta} \theta \leq \frac{\theta^{2}}{2}+\frac{\tilde{\theta}^{2}}{2}
\end{array}\right.
$$

Substituting (55) into (54), we get

$$
\begin{aligned}
\dot{V} \leq & s h(x, u, t) u+s H_{m}\left[\frac{s \hat{\theta}}{2 a^{2}} \phi^{T}(x) \phi(x)+\hat{\delta} \tanh \left(\frac{s}{\varsigma}\right)\right. \\
& \left.+H_{m}^{-1} \Phi \tanh \left(\frac{s \Phi}{\varsigma}\right)\right]+0.2785 \varsigma\left(H_{m} \delta+1\right)-\frac{v_{1} H_{m}}{2} \tilde{\delta}^{2} \\
& -\frac{v_{2} H_{m}}{2} \tilde{\theta}^{2}+H_{m}\left(v_{1} \frac{\delta^{2}}{2}+v_{2} \frac{\theta^{2}}{2}\right)+\frac{a^{2}}{2}
\end{aligned}
$$

Based on (56), the adaptive neural control law is designed as

$$
\begin{aligned}
u= & -k s-\frac{\hat{\theta} s}{2 a^{2}} \phi^{T}(x) \phi(x)-\hat{\delta} \tanh \left(\frac{s}{\varsigma}\right) \\
& -\xi \Phi \tanh \left(\frac{s \Phi}{\varsigma}\right)
\end{aligned}
$$

where $k>0$ and $\xi \geq H_{m}^{-1}$ are design parameters.

Remark 6: In reality, the term $-\frac{\hat{\theta} s}{2 a^{2}} \phi^{T}(x) \phi(x)$ of (57) is designed to eliminate the effect of unknown function $f(x, 0)$; the term $-\hat{\delta} \tanh \left(\frac{s}{S}\right)$ of (57) is designed to eliminate the effects of NN approximation error and external disturbance, which will enhance the robustness of the closed-loop control system; the term $-\xi \Phi \tanh \left(\frac{s \Phi}{S}\right)$ of (57) is designed to eliminate the effect of function $\Phi$ generated in the process of controller design. Therefore, the effects of the second term in the right side of (56) will be offset by the latter three terms of control law (57), and then the linear feedback term $-k s$ will be used to guarantee the fast convergence of tracking error.

By recalling Remark 5 , we have $\hat{\theta}(t) \geq 0, \hat{\delta}(t) \geq 0$ for $\forall t>0$ by choosing $\hat{\theta}(0) \geq 0, \hat{\delta}(0) \geq 0$, respectively. Moreover, it can be easily found that

$$
\left\{\begin{aligned}
\frac{s^{2}}{2 a^{2}} \phi^{T}(x) \phi(x) & \geq 0 \\
s \tanh \left(\frac{s}{\varsigma}\right) & \geq 0 \\
s \Phi \tanh \left(\frac{s \Phi}{\varsigma}\right) & \geq 0
\end{aligned}\right.
$$

Therefore, by substituting (57) into (56) and noting $h(x, u, t) \geq H_{m}>0$ and $\xi \geq H_{m}^{-1}$, we can rewrite (56) as

$$
\begin{aligned}
\dot{V} \leq & -k H_{m} s^{2}+0.2785 \varsigma\left(H_{m} \delta+1\right)-\frac{v_{1} H_{m}}{2} \tilde{\delta}^{2} \\
& -\frac{v_{2} H_{m}}{2} \tilde{\theta}^{2}+H_{m}\left(v_{1} \frac{\delta^{2}}{2}+v_{2} \frac{\theta^{2}}{2}\right)+\frac{a^{2}}{2}
\end{aligned}
$$

$\dot{V}$ will become negative by appropriately adjusting design parameters.

\subsection{Stability analysis}

In this subsection, the main results of this paper is stated in the following theorem, and the stability analysis of the closed-loop system is given.

Theorem 1: Consider the uncertain non-affine nonlinear system (1) under Assumption 1-3, the control law (57), and the adaptation laws (49), (50). Then, Given any $p>0$, for bounded initial conditions satisfying $\hat{\theta}(0) \geq 0, \hat{\delta}(0) \geq 0$ and $V(0) \leq p$, there exist $k, a, \varsigma$, $v_{1}, v_{2}, \gamma, \beta$ and $\xi$ such that

1) $V(t) \leq p$ for $\forall t>0$, and all the signals of closed-loop system are semi-globally uniformly ultimately bounded;

2) the state vector $x$ always remains in compact set $\Omega_{x}$ defined as

$$
\Omega_{x}=\left\{x(t) \mid\left\|x-x_{d}\right\| \leq \eta,\left[x_{d}, y_{d}^{(n)}\right]^{T} \in \Omega_{0}\right\}, \forall t \geq 0
$$


where $\eta$ is a positive constant depending on initial conditions and design parameters;

3 ) the tracking error $e$ will converge to the compact set $\Omega_{e}$, which is defined as

$$
\Omega_{e}=\left\{e|| e_{i} \mid \leq 2^{i-1} q^{i-n} B, \quad i=1,2, \ldots, n\right\}
$$

where $B$ is a positive constant depending on design parameters, which can be made arbitrarily small by appropriately adjusting design parameters. Therefore, the compact set $\Omega_{e}$ can be made arbitrarily small.

Proof: In this paper, we choose the Lyapunov function as (24), and therefore (59) can be rewritten as

$$
\dot{V} \leq-\mu V+\rho
$$

where

$$
\left\{\begin{array}{l}
\mu=\min \left\{2 k H_{m}, v_{1} \gamma, v_{2} \beta\right\} \\
\rho=0.2785 \varsigma\left(H_{m} \delta+1\right)+H_{m}\left(v_{1} \frac{\delta^{2}}{2}+v_{2} \frac{\theta^{2}}{2}\right)+\frac{a^{2}}{2}
\end{array}\right.
$$

It can be seen from (63) that $\rho / \mu$ can be made arbitrarily small by reducing $\varsigma, v_{1}, v_{2}, a$, and meanwhile, increasing $k, \beta, \gamma$. By choosing the appropriate design parameters, we can have $\rho / \mu \leq p$. Then, according to (62), we have that $\dot{V} \leq 0$ holds for $V=p$. Therefore, by the analysis of Remark 3 , we can know that $\Omega_{2}$ is an invariant set and the closed-loop system is stable. Thus, the property 1) of Theorem 1 is proven.

Multiplying (62) by $e^{\mu t}$, it becomes

$$
(\dot{V}(t)+\mu V(t)) e^{\mu t} \leq \rho e^{\mu t}
$$

Further, one has

$$
\frac{d}{d t}\left(V(t) e^{\mu t}\right) \leq \rho e^{\mu t}
$$

Integrating (65) over $[0, t]$, we get

$$
V(t) \leq(V(0)-\Gamma) e^{-\mu t}+\Gamma
$$

where $\Gamma=\rho / \mu$.

According to (66), we can know that $V(t)$ is bounded and therefore, $s, \tilde{\delta}, \tilde{\theta}$ is semi-globally uniformly ultimately bounded. From (24) and (66), we have

$$
\frac{1}{2} s^{2} \leq V(t) \leq V(0)+\Gamma
$$

Further, we have

$$
|s| \leq \sqrt{2(V(0)+\Gamma)}
$$

Define $\omega=\left[e_{1}, e_{2}, \ldots, e_{n-1}\right]^{T}$ and $\Lambda=\left[\lambda_{1}, \lambda_{2}, \ldots\right.$, $\left.\lambda_{n-1}\right]^{T}$, we can have

$$
\left\{\begin{array}{l}
s=\Lambda^{T} \omega+e_{n} \\
\dot{\omega}=A_{s} \omega+b_{s} s
\end{array}\right.
$$

where $A_{s}=\left[\begin{array}{cccc}0 & 1 & \cdots & 0 \\ \vdots & \vdots & \vdots & \vdots \\ 0 & 0 & \cdots & 1 \\ -\lambda_{1} & -\lambda_{2} & \cdots & -\lambda_{n-1}\end{array}\right], b_{s}=[0,0, \ldots$,

$1]^{T}$. Noting that $A_{s}$ is a Hurwitz matrix, hence, there exists a positive constant $c_{1}$ satisfying $\left\|e^{A_{s} t}\right\| \leq c_{1} e^{-\lambda_{1} t}$. From (69), we can get

$$
\omega(t)=e^{A_{s} t} \omega(0)+\int_{0}^{t} e^{A_{s}(t-\tau)} b_{s} s(\tau) d \tau
$$

Using (68), we have

$$
\begin{aligned}
\|\omega(t)\| & \leq c_{1}\|\omega(0)\| e^{-\lambda_{1} t}+c_{1} \int_{0}^{t} e^{-\lambda_{1}(t-\tau)}|s(\tau)| d \tau \\
& \leq c_{1}\|\omega(0)\|+\frac{c_{1} \sqrt{2(V(0)+\Gamma)}}{\lambda_{1}}
\end{aligned}
$$

Noting $s=\Lambda^{T} \omega+e_{n}$ and $e=\left[\omega^{T}, e_{n}\right]^{T}$, we have

$$
\|e\| \leq\|\omega\|+\left|e_{n}\right| \leq(1+\|\Lambda\|)\|\omega\|+|s|
$$

Substituting (68) and (71) into (72), we get

$$
\|e\| \leq \eta
$$

where

$$
\begin{aligned}
\eta= & c_{1}(1+\|\Lambda\|)\|\omega(0)\| \\
& +\left[1+\frac{(1+\|\Lambda\|) c_{1}}{\lambda_{1}}\right] \sqrt{2(V(0)+\Gamma)}
\end{aligned}
$$

and further we know that $\eta$ depends on the initial conditions and design parameters. Therefore, the property 2) of Theorem 1 is proven.

Furthermore, from (16), (24) and (66), we have

$$
\begin{aligned}
\lim _{t \rightarrow+\infty}|s| & \leq \lim _{t \rightarrow+\infty} \sqrt{2 V(t)} \\
& \leq \lim _{t \rightarrow+\infty} \sqrt{2\left[(V(0)-\Gamma) e^{-\mu t}+\Gamma\right]}
\end{aligned}
$$

Noting $\lim _{t \rightarrow+\infty}(V(0)-\Gamma) e^{-\mu t}=0$, therefore we have

$$
\lim _{t \rightarrow+\infty}|s| \leq B
$$

where $B=\sqrt{2 \Gamma}$. According to the property ii) of Lemma 4 , it is easy to get that the property 3 ) of Theorem 1 holds. In conclusion, $\Gamma$ can be made arbitrarily small by appropriately choosing design parameters. 
Thus, the compact set $\Omega_{e}$ can be made arbitrarily small. This completes the proof.

Remark 7: There are different design parameters that can have various effects on the performance of the proposed scheme. Note that adaptation gains $\gamma$ and $\beta$ in adaptation laws (49) and (50) that are used to tune the convergence rate of the adaptation process, and higher adaptation gains can make the convergence rate faster. Moreover, the design parameters $\varsigma$ and $a$ can also determine the convergence rate of the adaptation process and smaller $\varsigma$ and $a$ can lead to faster convergence. The small positive constants $v_{1}$ and $v_{2}$ are e-modification factors which can improve the stability of (49) and (50). The parameter $k$ is set to guarantee that tracking error $e$ converges to compact set $\Omega_{e}$ asymptotically in finite time and higher $k$ can lead to faster convergence. For design parameter $\xi \geq H_{m}^{-1}$, it does not influence the size of tracking error, and we can get its value by the method of trial and error in the simulation because of unknown positive constant $H_{m}$. It can be seen from the property 3 ) of Theorem 1 that the size of $\Omega_{e}$ is determined by $B=\sqrt{2 \Gamma}$, which can be decreased by reducing $\varsigma, v_{1}$, $v_{2}, a$, and meanwhile, increasing $k, \beta, \gamma$.

\section{Simulation}

In this section, two examples are given to illustrate the effectiveness of the proposed scheme. It is necessary to emphasize that external disturbance and parameter uncertainty are considered in two simulation examples.

Example 1: Consider the following third-order vander-Pol oscillator described by[25] :

$$
\left\{\begin{array}{l}
\dot{x}_{1}=x_{2} \\
\dot{x}_{2}=x_{3} \\
\dot{x}_{3}=f(x, u)+d(t) \\
y=x_{1}
\end{array}\right.
$$

where $d(t)=\sin (2 \pi t)$ is an external disturbance, and $f(x, u)$ is described as follows

$$
\begin{aligned}
f(x, u)= & -x_{1}-0.5 x_{2}+0.7\left(1-\left(x_{1}+0.5 x_{2}\right)^{2}\right) x_{3} \\
& +\left(r_{0}(x)+\Delta r(x)\right)\left(\psi(u)+\frac{1}{3} \psi(u)^{3}+\sin (\psi(u))\right)
\end{aligned}
$$

where $r_{0}(x)$ and $\Delta r(x)$ are the nominal part and the parameter variation part of uncertain parameter, respectively. To facilitate the simulation, $r_{0}(x)=2$ and $\Delta r(x)=\sin \left(x_{1} x_{2} x_{3}\right)$ are arranged.

It is worth noting that, for comparison purpose later, we choose $\psi(u)=u$ without dead-zone or backlash nonlinearities that will make $f(x, u)$ non-differentiable with respect to $u$. However, system (77) still satisfies Assumption 1. Furthermore, the desired trajectory $y_{d}=\sin (0.5 t)+\cos (t)$.

Based on Theorem 1, the adaptive neural tracking controller is designed as (57) and the adaptation laws are given as (49), (50). In this simulation, the design parameters are taken as $k=2, \varsigma=0.1, v_{1}=0.1$, $v_{2}=0.2, \lambda_{1}=\lambda_{2}=4, \gamma=5, \beta=4, \xi=1$ and $a=0.25$. The Gaussian RBF neural network $\hat{W}^{T} \phi(x)$ is constructed by using 27 nodes, with the centers $\mu_{i}$ evenly spaced in interval $[-4,4] \times[-4,4] \times[-4,4]$ and with width $\sigma_{i}=2.5$. The initial conditions are chosen as $\left[x_{1}(0), x_{2}(0), x_{3}(0)\right]^{T}=[0.5,0,0]^{T}, \hat{\delta}(0)=0$ and $\hat{\theta}(0)=0$. In the simulation, $d(t)$ is added in the closedloop system from $t=25 \mathrm{~s}$ to observe the effect of external disturbance. The simulation results are shown as Figs. 2-5.

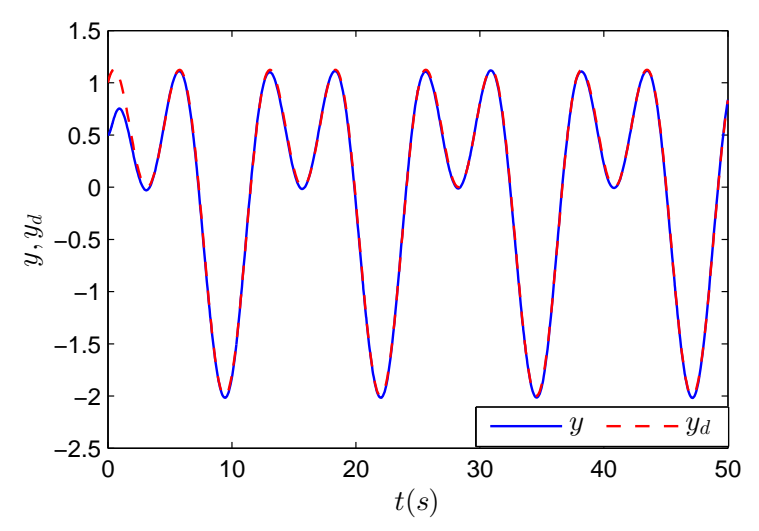

Figure 2: Output $y$ and desired trajectory $y_{d}$ for Example 1

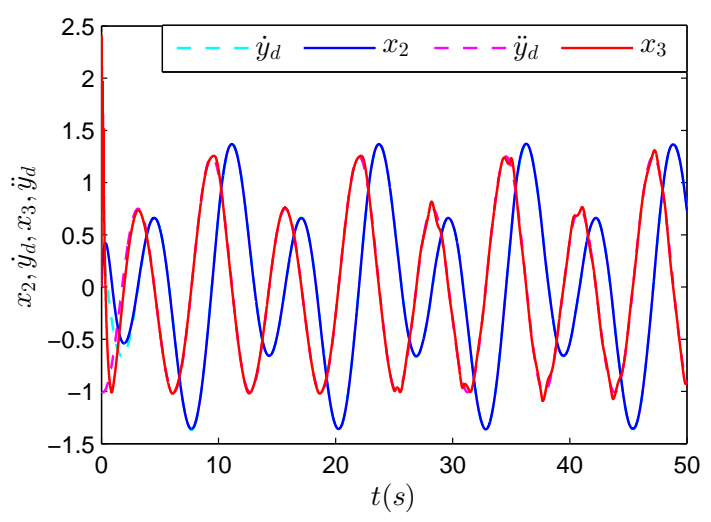

Figure 3: States $x_{2}, x_{3}$ and reference trajectories $\dot{y}_{d}, \ddot{y}_{d}$ for Example 


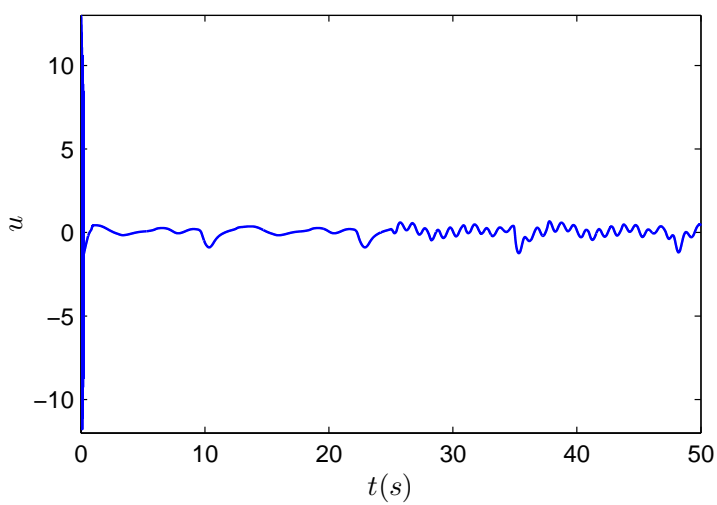

Figure 4: Control input $u$ for Example 1

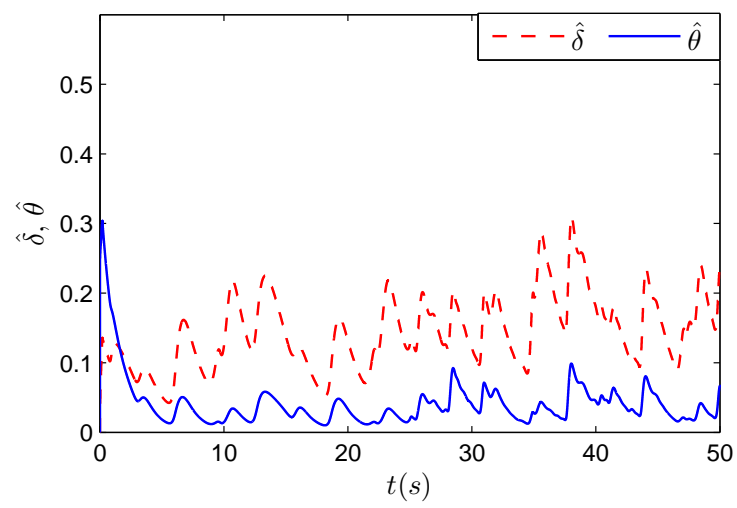

Figure 5: Adaptive parameters $\hat{\delta}$ and $\hat{\theta}$ for Example 1

It can be seen from Figs. 2-3 that fairly good tracking performance is obtained. The boundedness of control input $u$ is presented in Fig. 4. The response curves of adaptive parameters $\hat{\delta}, \hat{\theta}$ are depicted in Fig. 5. The simulation results demonstrate the excellent tracking capability of the proposed controller and its effectiveness for tracking control of non-affine nonlinear system (77).

Note that $f(x, u)$ is a smooth non-affine function in Example 1, and therefore, we can compare the response of the proposed approach with the direct adaptive neural control (DANC) approach in [24] to further testify the effectiveness of the proposed approach. The output responses under two methods are shown in Figs. 6 and 7. Moreover, the tracking performances of the two schemes are summarized in Table 1, where the max error is chosen as the max $\left|e_{1}\right|$, the average error is defined as $M e_{1}=\sum_{t=0}^{50}\left|e_{1}\right| / 50$ and the standard deviation is defined as $\sum_{t=0}^{50}\left(y-M e_{1}\right)^{2} / 50$. The results show that the proposed controller can track the desired trajectory as good as the DANC. However, only 2 adaptive param- eters are required by using MLP technique in the proposed method, and the DANC method needs 7 adaptive parameters at least, namely, the number of adaptive parameters used in the proposed method is much less than in the DANC method.

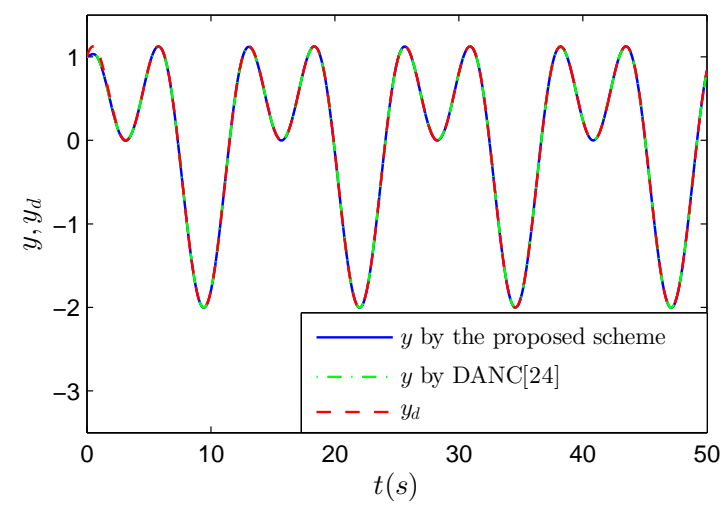

Figure 6: Output $y$ of two control schemes and desired trajectory $y_{d}$ with $\left(x(0)=[1,0,0]^{T}\right)$

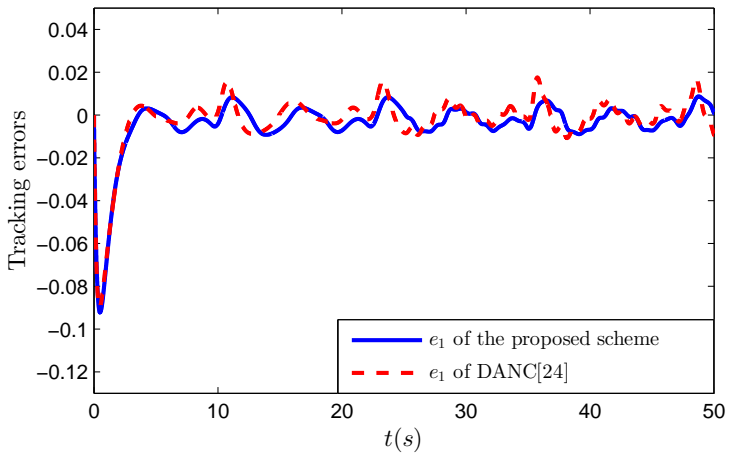

Figure 7: Tracking errors of two control schemes with $(x(0)=$ $\left.[1,0,0]^{T}\right)$

Table 1: Performance Comparison of Two Method

\begin{tabular}{cccc}
\hline \hline Methods & Max & Average & Standard deviation \\
\hline DANC $[24]$ & 0.0897 & 0.0074 & 0.0136 \\
\hline Proposed method & 0.0922 & 0.0073 & 0.0137 \\
\hline \hline
\end{tabular}

Remark 8: When dead-zone or backlash nonlinearities are considered in the control input, non-affine function $f(x, u)$ will be non-differentiable with respect to $u$. In this case, the DANC scheme is no longer applicable, but the proposed approach is still applicable. Therefore, the proposed approach can be applied to the non-affine nonlinear systems with non-affine function being possi- 
bly non-differentiable, which make the controller design more difficult. To further illustrate the applicability of the proposed scheme, the backlash nonlinearity is considered in Example 2.

Example 2: Consider the following continuous-time non-affine nonlinear system [26] :

$$
\left\{\begin{array}{l}
\dot{x}_{1}=x_{2} \\
\dot{x}_{2}=f(x, u)+d(t) \\
y=x_{1}
\end{array}\right.
$$

where $d(t)=\sin (2 \pi t)$ is an external disturbance, and $f(x, u)$ is described as follows

$$
\begin{aligned}
f(x, u) & =x_{1} x_{2}^{2}+x_{2} e^{-1-x_{1}^{2}}-x_{1} x_{2} \\
& +\cos (0.1 \psi(u))+\left(r_{0}(x)+\Delta r(x)\right) \psi(u)
\end{aligned}
$$

where $r_{0}(x)$ and $\Delta r(x)$ are defined similar as in Example 1. To facilitate the simulation, $r_{0}(x)=2$ and $\Delta r(x)=$ $0.3 \sin x_{2}^{2}$ are arranged. Furthermore, $\psi(u)$ is given as follows

$$
\psi(u)= \begin{cases}u-0.5 & \dot{u}>0 \text { and } \psi(u)=u-0.5 \\ u+0.5 & \dot{u}<0 \text { and } \psi(u)=u+0.5 \\ \psi\left(t_{-}\right) & \text {otherwise }\end{cases}
$$

where $u$ is the actual control input. It can be easily seen that the continuous non-affine function $f(x, u)$ is nondifferentiable with respect to $u$ because of the presence of backlash nonlinearity. However, system (79) satisfies Assumption 1. The desired trajectory $y_{d}=\sin (0.5 t)+$ $\cos (t)$.

Based on Theorem 1, the adaptive neural tracking control law is designed as (57) and the adaptation laws are given as (49), (50). In this simulation, the design parameters are taken as $k=2.5, \varsigma=0.25, v_{1}=0.15$, $v_{2}=0.4, \lambda_{1}=2, \gamma=5, \beta=3, \xi=1$ and $a=0.25$. The Gaussian RBF neural network $\hat{W}^{T} \phi(x)$ is constructed by choosing 9 nodes, with the centers $\mu_{i}$ evenly spaced in interval $[-4,4] \times[-4,4]$ and with width equal to 2 . The initial conditions are given as $\left[x_{1}(0), x_{2}(0)\right]^{T}=[0.5,0]^{T}, \hat{\delta}(0)=0$ and $\hat{\theta}(0)=0$. In the simulation, $d(t)$ is added in the closed-loop system from $t=25 \mathrm{~s}$ for the same aim as Example 1. The simulation results are shown in Figs. 8-11.

From Figs. 8-9, it is observed clearly that output $y$ and state $x_{2}$ converge rapidly to the reference signals $y_{d}$ and $\dot{y}_{d}$, respectively. As seen from Figs. 10-11, control input $u$ and adaptive parameters $\hat{\delta}, \hat{\theta}$ are bounded. The simulation results indicate that the proposed controller achieves good tracking performance even though there exist backlash nonlinearity and external disturbance in the non-affine nonlinear system (79).

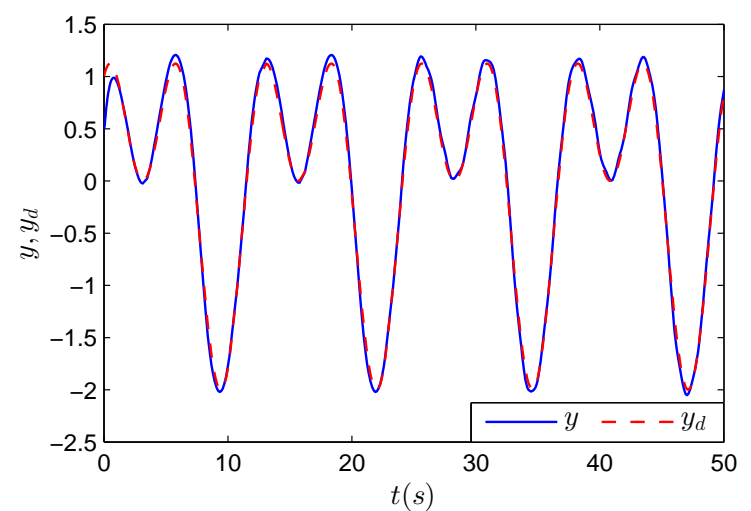

Figure 8: Output $y$ and desired trajectory $y_{d}$ for Example 2

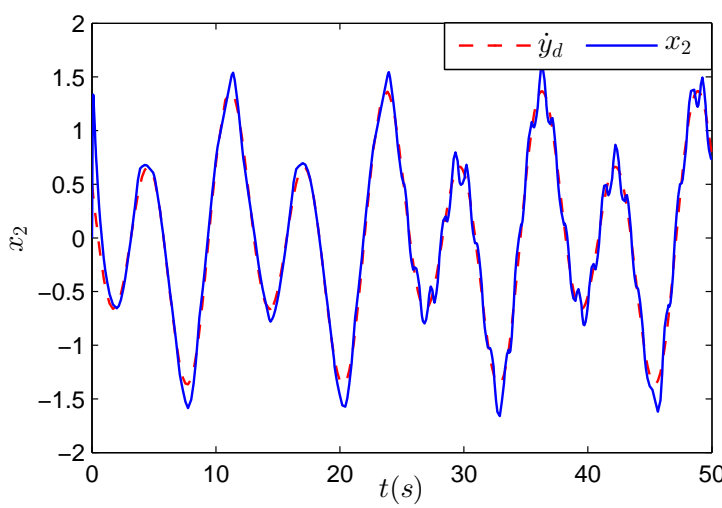

Figure 9: State $x_{2}$ and reference trajectory $\dot{y}_{d}$ for Example 2

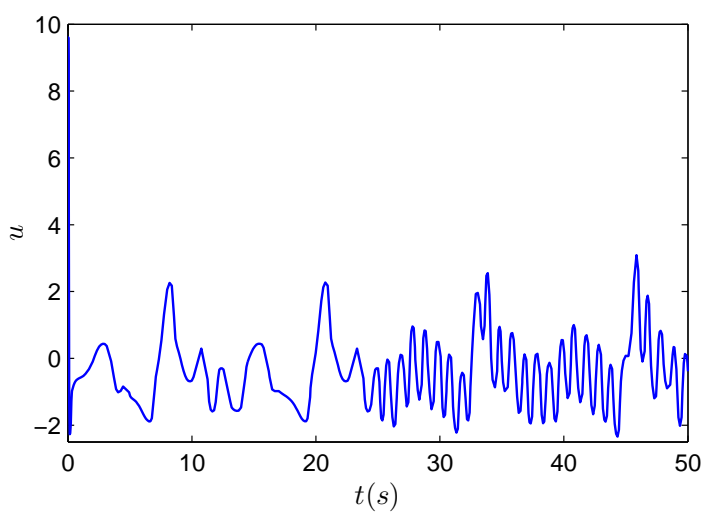

Figure 10: Control input $u$ for Example 2

To investigate the effect of different design parameters on the dynamic response of the system, three sets of design parameters are considered: 


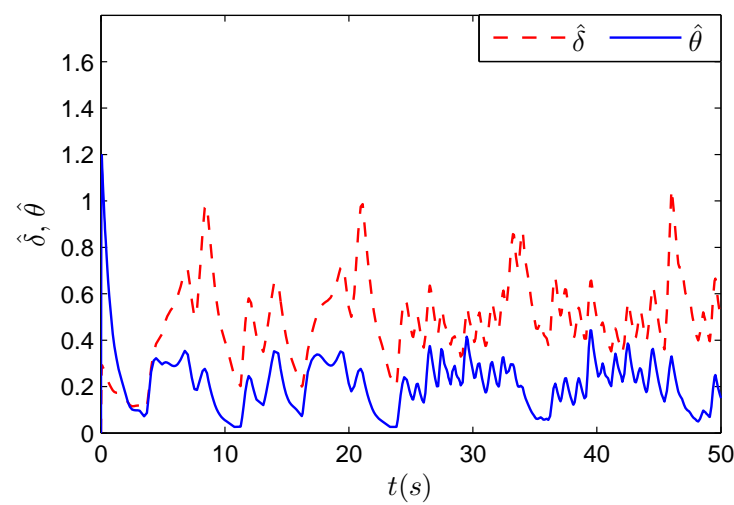

Figure 11: Adaptive parameters $\hat{\delta}$ and $\hat{\theta}$ for Example 2

$$
\begin{gathered}
I:\left\{\begin{array}{l}
\varsigma=0.35, v_{1}=0.25, v_{2}=0.5, a=0.35 \\
k=2, \beta=2.5, \gamma=4.5
\end{array}\right. \\
I I:\left\{\begin{array}{l}
\varsigma=0.25, v_{1}=0.15, v_{2}=0.4, a=0.25 \\
k=2.5, \beta=3, \gamma=5
\end{array}\right. \\
I I I:\left\{\begin{array}{l}
\varsigma=0.15, v_{1}=0.05, v_{2}=0.3, a=0.15 \\
k=3, \beta=3.5, \gamma=5.5
\end{array}\right.
\end{gathered}
$$

where the second set is from Example 2.

Based on the closed-loop control system of Example 2 , we conduct the simulation by using the three sets of design parameters. And the other parameters are the same as Example 2. By the last simulation, the output responses under the three sets are shown in Figs. 12 and 13. Moreover, the tracking performances are summarized in Table 2, in which the definition of max error, average error and standard deviation of output tracking error $e_{1}$ is the same as the ones of Table 1. Finally, the simulation results show that the compact set $\Omega_{e}$ can be made small by reducing $\varsigma, v_{1}, v_{2}, a$, and meanwhile, increasing $k, \beta, \gamma$. Therefore, the adjusting method of design parameters is validity.

Table 2: Output Tracking Error under Three Sets

\begin{tabular}{cccc}
\hline \hline Parameter sets & Max & Average & Standard deviation \\
\hline$I$ & 0.2025 & 0.0715 & 0.0591 \\
\hline$I I$ & 0.1306 & 0.0496 & 0.0418 \\
\hline$I I I$ & 0.0671 & 0.0271 & 0.0232 \\
\hline \hline
\end{tabular}

\section{Conclusion}

By modelling the non-affine function appropriately, a novel adaptive neural tracking control scheme has been

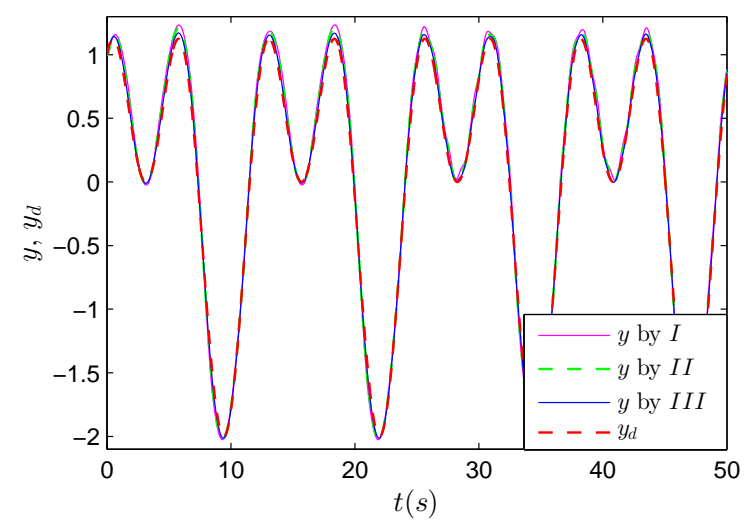

Figure 12: Output $y$ under 3 parameter sets and desired trajectory $y_{d}$ with $\left(x(0)=[1,0]^{T}\right)$

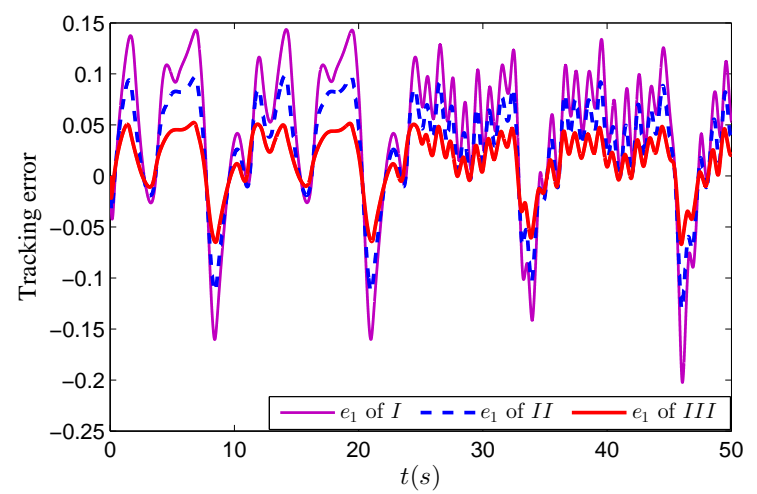

Figure 13: Tracking errors under 3 parameter sets with $(x(0)=$ $\left.[1,0]^{T}\right)$

presented for a more general class of uncertain nonaffine nonlinear system. The assumption on the nonaffine function is more relaxed as only a semi-bounded and continuous condition is required. The MLP technique has also been used to reduce the number of adaptive parameters, and the adaptive neural tracking controller has been designed based on the compact set. Finally, it is proven that this compact set is an invariant set for appropriate design parameters, which results in the stability of the closed-loop system. Therefore, the proposed controller can guarantee that all the signals in the closed-loop system are semi-global uniformly ultimately boundedness and the tracking error converges to a small residual set asymptotically. Finally, simulation resu lts have demonstrated the effectiveness of the proposed approach. 


\section{Acknowledgment}

This work was supported by National Science Foundation of China (Grant no. 61304120 and 61473307) and Aeronautical Science Foundation of China (Grant no. 20155896026). The authors are grateful to the editor and the anonymous reviewers for constructive comments that helped to improve the quality and presentation of this paper.

[1] C.Y. Lee, J.J. Lee, Adaptive control for uncertain nonlinear systems based on multiple neural networks, IEEE Trans. Syst. Man Cybern. Part B: Cybern. 34 (1) (2004) 325-333.

[2] S.S. Ge, C. Wang, Adaptive neural control of uncertain MIMO nonlinear systems, IEEE Trans. Neural Netw. 15 (3) (2004) 674692.

[3] S.C. Tong, Y.M. Li, G. Feng, T.S. Li, Observer-based adaptive fuzzy back-stepping dynamic surface control for a class of MIMO nonlinear systems, IEEE Trans. Syst. Man Cybern. Part B: Cybern. 41 (4) (2011) 1124-1135.

[4] A. Boulkroune, A. Bouzeriba, S. Hamel, T. Bouden, A projective synchronization scheme based on fuzzy adaptive control for unknown multivariable chaotic systems, Nonlinear Dyn. 78 (1) (2014) 433-447.

[5] Z.C. Liu, X.M. Dong, J.P. Xue, Y. Chen, Adaptive neural control for a class of time-delay systems in the presence of backlash or dead-zone nonlinearity, IET Control Theory Appl. 8 (11) (2014) 1009-1022.

[6] H.L. Dong, Z.D. Wang, F.E. Alsaadi, B. Ahmad, Eventtriggered robust distributed state estimation for sensor networks with state-dependent noises, Int. J. Gen. Syst. 44 (2) (2015) 254266.

[7] K. Esfandiari, F. Abdollahi, H.A. Talebi, Adaptive control of uncertain nonaffine nonlinear systems with input saturation using neural networks, IEEE Trans. Neural Netw. Learn. Syst. 26 (10) (2015) 2311-2322.

[8] C.C. Hua, X.P. Guan, P. Shi, Robust output feedback tracking control for time-delay nonlinear systems using neural network, IEEE Trans. Neural Netw. 18 (2) (2007) 495-505.

[9] H. Nan, H.L. Dong, Z.D. Wang, W.J. Ren, F.E. Alsaadi, Nonfragile state estimation for discrete Markovian jumping neural networks, Neurocomputing 179 (2016) 238-245.

[10] M. Wang, X.P. Liu, P. Shi, Adaptive neural control of purefeedback nonlinear time-delay systems via dynamic surface technique, IEEE Trans. Syst. Man Cybern. Part B: Cybern. 41 (6) (2011) 1681-1692

[11] Y.J. Yu, H.L. Dong, Z.D. Wang, W.J. Ren, F.E. Alsaadi, Design of non-fragile state estimators for discrete time-delayed neural networks with parameter uncertainties, Neurocomputing 182 (2016) $18-24$

[12] S.S. Ge, C.C. Hang, T. Zhang, Nonlinear adaptive control using neural networks and its application to CSTR systems, J. Process Control 9 (4) (1999) 313-323.

[13] A.S. Shiriaev, H. Ludvigsen, O. Egeland, A.L. Fradkov, Swinging up of non-affine in control of pendulum, in: Proc. of the American Control Conference (1999) 4039-3044.

[14] B.J. Yang, A.J. Calise, Adaptive control of a class of nonaffine systems using neural networks, IEEE Trans. Neural Netw. 18 (4) (2007) 1149-1459.

[15] N. Hovakimyan, B.J. Yang, A.J. Calise, Adaptive output feedback control methodology applicable to non-minimum phase nonlinear systems, Automatica 42 (4) (2006) 513-522.

[16] A.J. Calise, N. Hovakimyan, M. Idan, Adaptive output feedback control of nonlinear systems using neural networks, Automatica 37 (8) (2001).

[17] J. Wang, S.S. Ge, T.H. Lee, Adaptive fuzzy sliding mode control of a class of nonlinear systems, in: Proc. of the Third Asian Control Conference (2000) 599-604.

[18] A. Boulkroune, M. M'Saad, M. Farza, Adaptive fuzzy tracking control for a class of MIMO nonaffine uncertain systems, Neurocomputing 93 (2012) 48-55.

[19] S. Labiod, T.M. Guerra, Indirect adaptive fuzzy control for a class of nonaffine nonlinear systems with unknown control directions, International Journal of Control, Automation, and Systems 8 (4) (2010) 903-907.

[20] S.S. Ge, J. Zhang, Neural-network control of nonaffine nonlinear system with zero dynamics by state and output feedback, IEEE Trans. Neural Netw. 14 (4) (2003) 900-918.

[21] H.G. Han, W.D. Zhou, J.F. Qiao, G. Feng, A direct selfconstructing neural controller design for a class of nonlinear systems, IEEE Trans. Neural Netw. Learn. Syst. 26 (6) (2015) 1312-1322.

[22] S. Labiod, T.M. Guerra, Adaptive fuzzy control of a class of SISO nonaffine nonlinear systems, Fuzzy Sets Syst. 158 (10) (2007) 1126-1137.

[23] T. Zhang, S.S. Ge, C.C. Hang, Direct adaptive control of nonaffine nonlinear system using multilayer neural networks, in: Proc. of the American Control Conference (1998) 515-519.

[24] M. Chen, S.S. Ge, Direct adaptive neural control for a class of uncertain nonaffine nonlinear systems based on disturbance observer, IEEE Trans. Cybern. 43 (4) (2013) 1213-1225.

[25] S.L. Dai, C. Wang, M. Wang, Dynamic learning from adaptive neural network control of a class of nonaffine nonlinear systems, IEEE Trans. Neural Netw. Learn. Syst. 25 (1) (2014) 111-123.

[26] H.B. Du, X.C. Chen, NN-based output feedback adaptive variable structure control for a class of non-affine nonlinear systems a nonseparation principle design, Neurocomputing 72 (7) (2009) 2009-2016

[27] B. Karimi, M.B. Menhaj, Non-affine nonlinear adaptive control of decentralized large-scale systems using neural networks, Inf. Sci. 180 (17) (2010) 3335-3347.

[28] Q.K. Shen, T.P. Zhang, Novel design of adaptive neural network controller for a class of non-affine nonlinear systems, Commun. Nonlinear Sci. Numer. Simul. 17 (3) (2012) 1107-1116.

[29] K. Esfandiari, F. Abdollahi, H. A. Talebi, Stable adaptive output feedback controller for a class of uncertain non-linear systems, IET Control Theory Appl. 9 (9) (2015) 1329-1337.

[30] A. Boulkroune, M.M'Saad, M. Tadjine, M. Farza, Adaptive fuzzy control for MIMO nonlinear systems with unknown deadzone, in: Proc. of the 4th International IEEE Conference on Intelligent Systems 1 (2008) 50-55.

[31] A. Boulkroune, M.M'Saad, M. Farza, State and output feedback fuzzy variable structure controllers for multivariable nonlinear systems subject to input nonlinearities, Int. J. Adv. Manuf. Technol. 71 (1-4) (2014) 539-556.

[32] Z.C. Liu, X.M. Dong, J.P. Xue, L.P. Zhang, Adaptive tracking control for nonlinear systems with a class of input nonlinearities, Asian J. Control 18 (2) (2016) 771-778.

[33] W. Yang, S.C. Tong, Robust stabilization of switched fuzzy systems with actuator dead-zone, Neurocomputing 173 (2016) 1028-1033.

[34] K.R. Chen, J.W. Wang, Y. Zhang, Z. Liu, Adaptive consensus of nonlinear multi-agent systems with unknown backlash-like hysteresis, Neurocomputing 175 (2016) 698-703.

[35] J. Xiang, Y. Li, W. Wei, Stabilisation of a class of non-affine systems via modelling error compensation, IET Control Theory Appl. 2 (2) (2008) 108-116.

[36] Y.H. Li, S. Qiang, X.Y. Zhuang, et al., Robust and adaptive 
backstepping control for nonlinear systems using RBF neural networks, IEEE Trans. Neural Netw. 15 (3) (2004) 693-701.

[37] H.G. Zhang, Y.H. Xie, C.X. Wang, et al., Adaptive synchronization between two different chaotic neural networks with time delay, IEEE Trans. Neural Netw. 18 (6) (2007) 1841-1845 .

[38] W.J. Dong, Y.Y. Zhao, Y.M. Chen, J.A. Farrell, Tracking control for nonaffine systems a self-organizing approximation approach, IEEE Trans. Neural Netw. Learn. Syst. 23 (2) (2012) 223-235.

[39] T.S. Li, D. Wang, G. Feng, S.C. Tong, A DSC approach to robust adaptive NN tracking control for strict-feedback nonlinear systems, IEEE Trans. Syst. Man Cybern. Part B: Cybern. 40 (3) (2010) 915-927.

[40] M. Wang, S.S. Ge, K.S. Hong, Approximation-based adaptive tracking control of pure-feedback nonlinear systems with multiple unknown time-varying delays, IEEE Trans. Neural Netw. 21 (11) (2010) 1804-1816.

[41] M.M. Polycarpou, P.A. Ioannou, A robust adaptive nonlinear control design, Automatica 32 (3) ( 1996) 423-427.

[42] H. Deng, M. Kristić, Stochastic nonlinear stabilization, part I: a backstepping design, Syst. Control Lett. 32 (3) (1997) 143-150.

[43] R.M. Sanner, J.J.E. Slotine, Gaussian networks for direct adaptive control, IEEE Trans. Neural Netw. 3 (6) (1992) 837-863.

[44] T. Zhang, S.S. Ge, C.C. Hang, Stable adaptive control for a class of nonlinear systems using a modified Lyapunov function, IEEE Trans. Autom. Control 45 (1) (2000) 129-132.

[45] D. Swaroop, J.K. Hedrick, P.P. Yip, J.C. Gerdes, Dynamic surface control for a class of nonlinear systems, IEEE Trans. Autom. Control 45 (10) (2000) 1893-1899. 